\title{
MÉTAPHORES JOURNALISTIQUES
}

\author{
Jean-Pierre Meunier ${ }^{1}$
}

Si l'on adopte à leur égard une perspective d'inspiration cognitiviste, les médias apparaissent comme un vaste dispositif de communication de connaissances, savoirs, idées, représentations. On le sait depuis longtemps comme le montre la formule déjà ancienne par laquelle on tente généralement de synthétiser les fonctions exercées pas les médias: informer, éduquer, divertir. Dans une telle formule, cependant, le terme "éduquer", outre qu'il comporte une connotation très didactique, très scolaire, semble n'évoquer qu'une fonction bien différenciée et localisée dans des genres relativement bien définis: les documentaires, certains reportages, les émissions de vulgarisation scientifique, certains débats, etc.

Éduquer, selon la formule classique, serait autre chose qu'informer. Cependant, si la fonction d'éduquer s'affirme clairement dans certains types de messages médiatiques, elle n'est évidemment pas absente des autres types, mais le reconnaitre suppose que l'on modifie quelque peu les conceptions -largement héritées de l'univers scolaireet de l'éducation et du savoir.

Tous les médias et tous les messages ont quelque chose à voir avec le savoir. Il faut reconnaître, dans la communication médiatique, -plus ou moins enchevêtrée avec d'autres fonctions et plus ou moins 1 Professeur à l'Université catholique de Louvain. Président du Département de
communication. 
saillante selon les divers types de messages- une fonction générale de communication des savoirs.

Informer -au sens journalistique du terme-implique qu'à travers les événements -d'actualité ou non-dont on parle, soient véhiculées des connaissances ou représentations des domaines de référence des événements en question: domaine historique, ou politique, ou économique, ou sportif... On aurait tort de sous-estimer cette fonction ou de la confiner -comme le fait la formule classique évoquée plus hautà un sous-secteur de la communication médiatique poursuivant à l'écran ou dans les colonnes des journaux et revues, l'œuvre d'éducation entreprise par l'école.

Aujourd'hui, la plupart des connaissances que possèdent les individus sur les sciences et les techniques, l'astronomie, la médecine et la santé, la géographie, la météorologie, l'histoire récente, les systèmes politiques, les mécanismes économiques, etc., ne proviennent précisément pas de l'école mais des médias.

A moins qu'ils ne fassent des études spécifiques en économie, la plupart des individus ne peuvent se former une représentation de notions telles que concurrence, compétitivité, croissance, PNB, etc., qu'à travers la masse des informations que diffusent quotidiennement les médias. Or, c'est avec de tels savoirs que ces mêmes individus se représentent leur environnement -naturel, social, politique...- et prennent -ou ne prennent pas- des décisions concernant leur vie quotidienne, individuelle et sociale. On voit dès lors se dessiner une nouvelle problématique que l'on peut supposer d'un certain intérêt pour le chercheur et pour le praticien soucieux de réfléchir sa pratique.

\section{Une nouvelle problématique}

Une nouvelle problématique, c'est-à-dire un nouveau centre d'attention, un nouveau point de focalisation pour le regard.

Une problématique, bien sûr, n'est jamais radicalement nouvelle. Il existe déjà une référence au savoir dans de nombreuses recherches -celles concernant les idéologies, par exemple, ou celles issues de l'intérêt actuel des chercheurs pour la réception-mais cette référence y reste malgré tout marginale. La nouveauté, en matière de problématique, surgit lorsque le marginal devient central, entraînant une nouvelle mise en perspective des phénomènes, et par suite de nouvelles questions en appelant elles-mêmes à de nouvelles notions. 
On ne saurait ici développer en détail la nouvelle problématique dont il est question -du reste, en grande partie à constituer- mais on peut évoquer quelques-unes des questions qu'elle suscite ou suscitera.

La centration sur la communication des savoirs fait surgir une série de questions sur les savoirs eux-mêmes. Qu'est-ce que le savoir? $\mathrm{Y}$ a-t-il des distinctions à faire entre les diverses notions plus ou moins similaires que l'on utilise pour le nommer: représentation, connaissance, savoir ...?

Une autre série de questions concerne le domaine des représentations mentales, domaine que les sciences humaines n'abordent qu'avec retenue du fait que son approche nécessite souvent un recours à des méthodes suspectées de subjectivisme ${ }^{1}$.

Quelles sont les représentations mentales et les opérations cognitives en jeu dans la communication en général?2 Plus particulièrement, quelles sont les représentations mentales et les opérations cognitives en jeu dans la communication médiatique? Comment se forment ces représentations? Quel usage les spectateurs ou lecteurs peuvent-ils en faire? Comment s'organisent-elles entre elles dans leur esprit? Pour parler plus précis encore, quelles formes mentales prennent les notions dont nous disions plus haut qu'elles proviennent pour la plupart des gens des médias? Comment se représente-t-on par exemple la notion économique de "compétitivité" et quel raisonnement peut-on fonder sur cette représentation? Comment cette représentation se relie-t-elle à celles de profit, salaire, échanges internationaux, nationalisation, etc.? En séquence, comme dans les traités d'économie? En réseau?

La recherche d'une réponse à ces questions oblige les chercheurs en communication à recourir systématiquement aux notions formées et explorées dans le cadre de la psychologie cognitive, ce qui, soit dit en passant, illustre d'une nouvelle manière le caractère nécessairement interdisciplinaire des recherches en communication.

Il va de soi que des notions comme celle d'image mentale et, plus particulièrement, de modèle mental peuvent être d'une grande utilité

1 L'introspection et la description phénoménologique. De telles méthodes cependant font actuellement l'objet d'un regain d'intérêt et d'un nouvel examen; cf, par exemple le livre de F. VARELA, E. THOMSON, E. RosCH, L'inscription corporelle de l'esprit, Sciences cognitives et expérience humaine, trad. franç., Paris, Seuil, 1993.

2 On sait que le livre de Dan SPERBER et Deirdre WILSON, La pertinence, communication et cognition, Paris, Éd. de Minuit, 1989, tourne tout entier autour de ces questions. 
dans l'approche des formes et fonctionnements des savoirs acquis par les grands moyens de communication.

Une autre série de questions, en rapport évidemment avec celles qu'on vient d'évoquer, concernent les médias eux-mêmes et les mises en formes qu'ils effectuent des savoirs, compte tenu des formes sémiotiques et narratives dont ils disposent ou qu'ils ont privilégiées, compte tenu aussi des contraintes qu'ils subissent du fait de leur insertion dans un certain contexte social, culturel, économique...

N'évoquons ici que l'une ou l'autre de ces questions. A quel type de représentation mentale et d'opération cognitive correspondent les différents langages utilisés par les médias, et leurs combinaisons? En particulier, à quel type de représentation et d'opération mentale correspond l'image, ce mode de communication privilégié par les médias? Que la communication médiatique soit avant tout iconique, cela ne signifie pas seulement -ce qui a été souvent noté- qu'elle privilégie le spectacle, cela signifie aussi qu'elle privilégie certains types d'imageries mentales et de mises en relation des éléments ${ }^{1}$. Quels types de représentations mentales et d'opérations cognitives suscitent les mises en récits des événements pratiqués par les médias? Par exemple est-ce la même chose de prendre connaissance de la politique à travers la narration des affrontements des politiciens ou à travers l'explication des problèmes à résoudre et l'exposé des choix possibles et de leurs conséquences éventuelles.

Une autre série de questions concerne les rapports qu'il peut y avoir entre la manière de présenter les contenus d'information -dans un langage plus technique: les dispositifs d'énonciation- et la façon dont les destinataires vont intégrer et maîtriser, utiliser ces contenus. Par exemple, quel usage du savoir peut faire le spectateur d'émissions de vulgarisation scientifique conçues sur un mode résolument didactique? Ou le spectateur de reportages dont les commentaires résolument assertifs inhibent tout autre interprétation que celles qu'ils imposent avec autorité?

D'autres questions possibles concernent les contraintes que les contextes économiques, politiques, culturels, font peser sur les mises en formes opérées par les médias.

Toutes ces questions sont complexes. Celles qu'on vient d'évoquer relèvent un peu de la caricature à des fins pédagogiques. Leur

1 Les langages sont des "technologies de l'intelligence", comme l'ont bien noté des auteurs comme J. GOODY ou P. LEVY. 
approche, on l'a déjà dit, réclame une interdisciplinarité difficile à réaliser. Une chose relativement facile est d'étudier les langages comme systèmes relativement isolables, autre chose est de les relier à la cognition avec toutes les difficultés méthodologiques que cela implique.

L'enjeu de cette démarche est cependant évident. Il ne s'agit pas seulement de notre connaissance de la communication médiatique -laquelle resterait tronquée si elle évitait d'aborder de front le domaine mental des représentations. Il s'agit aussi de politique et d'éthique. La connaissance de la connaissance communiquée par les médias débouche fatalement sur cette question: cette connaissance est-elle à même d'augmenter la capacité des individus à maîtriser et à se frayer une voie dans leur environnement naturel, social, politique, etc.? Va-t-elle ou non dans le sens de ce que Morin appelait, d'une expression parlante, la "démocratie cognitive"?

\section{Métaphore et cognition}

Il semble évident que la notion de métaphore soit amenée à occuper une place proche du centre dans la problématique que l'on vient d'évoquer. La métaphore s'observe partout dans les médias. On peut l'observer dans tous les langages qu'ils utilisent: langage des mots bien sûr, mais aussi langage des images (fixes ou animées), langage des sons. On peut d'ailleurs en dire autant de la métonymie, cette autre figure majeure de la rhétorique. Métaphore et métonymie traversent tous les discours médiatiques: ceux qui ont pour objectif d'informer ou d'apprendre autant que ceux qui -comme le discours publicitaire- ont pour but de persuader.

Or, on sait maintenant que métaphore et métonymie ne sont pas que jeux de mots, que la notion rhétorique de figure de style n'en épuise pas le sens. On sait notamment que ces notions comportent une importante dimension cognitive. Pour Lakoff et Johnson, cette dimension occupe tout l'avant-plan: la métaphore est avant tout conceptuelle et dépendrait d'autant moins du jeu avec les mots que ce serait plutôt celui-ci qui dépendrait d'elle.

"L'hypothèse la plus importante que nous avons émise jusqu'ici est donc que la métaphore n'est pas seulement affaire de langage ou question de mots. Ce sont au contraire des proces- 
sus de pensée humaine qui sont en grande partie métaphoriques. C'est ce que nous voulons dire quand nous disons que le système conceptuel humain est structuré et défini métaphoriquement. Les métaphores dans le langage sont possibles précisément parce qu'il y a des métaphores dans le système conceptuel de chacun"1.

On voit la différence de point de vue par rapport à d'autres perspectives plus classiques comme celle encore récente du structuralisme. Essentiellement centré sur le(s) langage(s) comme système(s) autonome(s), celui-ci ne pouvait concevoir la métaphore autrement que comme jeu avec les mots et les significations: substitution de signifiants, à partir d'une ressemblance -ou d'une contiguïté pour la métonymie- au niveau du signifié, constitution consécutive d'un sens propre et d'un sens figuré2.

La perspective adoptée par Lakoff et Johnson procède d'un déplacement de l'attention du langage vers la pensée, laquelle, c'est important de le noter, est conçue sur un mode phénoménologique faisant grand cas de l'expérience vécue. Par pensée, il faut comprendre notre capacité de compréhension telle qu'elle se fonde sur notre corporéité et s'alimente à notre culture. Le corps, l'environnement physique et l'environnement culturel donnent une structure à notre expérience.

"Nous comprenons directement notre expérience quand nous concevons qu'elle est structurée de manière cohérente en termes de Gestalts qui ont émergé directement de l'interaction avec (et dans) notre environnement"3.

La métaphore, dès lors, émerge d'une superposition dans l'expérience plutôt que dans les mots.

"L'essence d'une métaphore est qu'elle permet de comprendre quelque chose (et d'en faire l'expérience) en termes de quelque chose d'autre" 4 .

et

1 George LakofF et Mark Johnson, Les métaphores dans la vie quotidienne, Paris, Éd. de Minuit, 1983, p. 16.

2 Pour certains auteurs structuralistes, la centration sur le langage est telle que l'opération qu'on vient de décrire se trouve inversée: la substitution de signifiants est première et les effets se sens dérivés.

3 Ibidem, p. 232.

4 Ibidem, p. 25. 
"Nous comprenons l'expérience de manière métaphorique quand nous utilisons une Gestalt appartenant à un domaine d'expérience pour structurer notre expérience dans un autre domaine"1.

Un exemple. Dans notre culture, l'expérience de la discussion est conçue au moins partiellement selon l'expérience de la guerre. C'est ce qui explique la présence, dans le langage quotidien, d'expressions telles que: "Vos affirmations sont indéfendables. Il a attaqué chaque point faible de mon argumentation (...)"2. Et ce n'est pas seulement la compréhension qui est en cause, c'est aussi l'action. Comprendre une expérience dans les termes d'une autre implique que l'on se comporte de manière partiellement similaire dans chacune d'elles.

De fait, dans notre culture, la discussion et l'argumentation sont toujours vécues sur le mode de l'affrontement.

"La discussion n'est certes pas une sous-espèce de la guerre: les discussions et les guerres sont deux types de choses différentes - le discours verbal et le conflit armé- et les actes effectués sont différents. Mais la discussion est partiellement structurée, comprise, pratiquée et commentée en termes de guerre"3.

Pour être complet, signalons aussi la conception cognitive de la métonymie proposée par les mêmes auteurs:

"La métonymie (...) a avant tout une fonction référentielle: elle nous permet d'utiliser une entité pour tenir lieu d'une autre. Mais la métonymie n'est pas seulement un instrument référentiel; elle a aussi pour fonction de faciliter la compréhension. Par exemple, dans le cas de la métonymie LA PARTIE POUR LE TOUT, bien des parties peuvent tenir lieu du tout. La partie que nous retenons détermine l'aspect du tout sur lequel nous nous concentrons" 4 .

Remarque importante: les deux conceptions de la métaphore que l'on vient d'examiner et d'opposer ne sont pas forcément exclusives. Nos pratiques d'intellectuels et de chercheurs nous obligent, souvent à des fins d'argumentation précisément (la discussion, c'est la guerre), à

\footnotetext{
1 lbidem, p. 242.

2 Ibidem, p. 14.

3 Ibidem, p. 15.

4 Ibidem, p. 45.
} 
des choix binaires en matière de théorie. Or, les théories -opposéesne font souvent que mettre en profil des aspects différents des choses et sans doute vaut-il mieux, plutôt que se comporter à leur égard selon l'alternative adoption ou rejet, de les composer. Il n'y a pas beaucoup de place ici pour justifier ce point de vue. On aimerait cependant, concernant la métaphore, formuler quelques propositions allant dans ce sens.

Premièrement, entre la pensée conçue comme expérience et les mots, la relation peut être considérée comme circulaire et non comme linéaire (ce qui est le cas, grosso modo, des deux perspectives théoriques examinées). Cependant, -c'est notre deuxièmement-, la circularité n'empêche pas que l'un ou l'autre des pôles soit prédominant. Il est des métaphores qui relèvent plus du jeu avec les mots et d'autres qui s'enracinent plus profondément dans l'expérience vécue des choses. Il nous semble possible d'imaginer une sorte de continuum entre les deux pôles.

Troisièmement, selon que la métaphore se rapproche de l'un ou l'autre pôle, ses fonctions et ses effets sont variables. Ce qu'on veut suggérer ici, c'est que non seulement la théorie met en relief certains aspects des choses au détriment d'autres, mais encore que ces aspects peuvent être plus ou moins réalisés dans les phénomènes observés.

Nous allons tenter d'illustrer ces propositions au moyen d'exemples empruntés aux médias.

\section{Métaphores médiatiques}

Soit une expression comme "Les écueils du dernier acte yougoslave" où l'on perçoit plusieurs métaphorisations: le mot "acte" nous invite à percevoir la guerre civile yougoslave comme une pièce de théâtre tandis que le mot "écueil", plus proche du domaine de la navigation que de celui des faits liés au règlement de la guerre, transpose dans celui-ci le sentiment des périls (écueil = "rocher à fleur d'eau", dit le petit Larousse) propre au premier. Ces métaphorisations modulent évidemment la connaissance que l'on prend des événements. Mais les domaines qu'elles superposent restent en même temps bien séparés. La métaphore -du reste relativement éculée- est ici occasionnelle; elle vise, à la faveur de l'analogie établie par le jeu de mots, à faire percevoir certains aspects d'une certaine guerre. Elle ne semble pas impliquer une structuration en profondeur de l'expérience 
et de l'action. Cela paraît encore plus vrai pour une expression telle que "Le scénario noir de la flotte rouge" dans laquelle le jeu de mots -que l'on peut vraiment nommer ici figure de style- apparaît presque pur, n'ayant presque plus d'autre effet que celui d'étonner, d'amuser, de séduire. Sa visée est surtout relationnelle: elle cherche à accrocher l'attention du lecteur et à l'entraîner dans une relation de complicité. "Une métaphore créative et réussie, écrivent Sperber et Wilson, révèle et souvent communique, outre son contenu propre, l'existence d'une certaine communion de pensée entre les interlocuteurs"1.

Mais que dire maintenant d'un énoncé tel que "un Japonais à la tête de la société $X$ " qui n'est presque plus une image, qui ne fait presque plus rien comprendre et n'établit aucune relation particulière. Il n'y a presque plus de jeu avec les mots parce que les domaines concernés -l'organisme vivant avec sa tête et l'organisation avec sa direction-sont dans notre expérience confondus au point que les mots relatifs à un domaine valent purement et simplement pour l'autre domaine. L'usage des mots témoigne ici d'une analogie profondément inscrite dans notre culture, dans notre vision partagée des choses et dans les pratiques qu'entraîne cette vision. Il nous est difficile de concevoir une organisation sociale autrement qu'avec une tête directrice, un cerveau, et réciproquement, il nous est difficile de concevoir ce dernier autrement que comme un organe de commande.

La conception de Lakoff et Johnson prend ici toute sa pertinence: on voit clairement le lien entre la métaphore et la structuration de l'expérience. Mais on voit aussi, par comparaison de différentes sortes de métaphores possibles -comparaison que nos exemples n'ont fait que suggérer- que la métaphore peut concerner la pensée de différentes manières ou à différents niveaux. Curieusement, quand on y réfléchit bien, l'énoncé "les écueils du dernier acte yougoslave" peut provoquer davantage la réflexion que "un Japonais à la tête de la société X". Même le (presque) pur jeu verbal "le scénario noir de la flotte rouge" paraît plus apte à déclencher la réflexion. C'est qu'il subsiste une distance suffisante entre le théâtre et la guerre, entre l'univers du film noir et la flotte rouge, distance qui se maintient à l'intérieur même de la superposition conceptuelle et oblige le lecteur lui-même à prendre du recul par rapport à la comparaison. C'est dans la mesure où la guerre ne peut être tout à fait vécue comme une pièce

1 Deirdre Wilson et Dan Sperber, "Ressemblance et communication", in Introduction aux Sciences cognitives, Gallimard, Folio Essais, 1992, p. 237-238. 
de thêâtre que, tout à la fois, nous sommes conscients de la comparaison et pouvons considérer l'éclairage conceptuel que cette comparaison ou superposition induit. Si au contraire la Gestalt expérientielle "guerre" en venait à recouvrir davantage la Gestalt "théâtre" (hypothèse rendue parfaitement plausible par les raffinements de la mise en spectacle de la guerre effectuée par les médias), il n'y aura plus guère de comparaison -décentration pourrait-on mieux dire- ni donc d'éclairage conceptuel, il y aura fixation conceptuelle. C'est lorsque le jeu avec les mots est manifeste que la réflexion peut être sollicitée. Lorsque le jeu avec les mots s'estompe, c'est que s'estompent aussi les différences et donc les possibilités de décentration et de réflexion.

Cette longue remarque, que l'on ne conduira pas ici plus avant, montre en tout cas la complexité des rapports entre métaphore et cognition. Elle montre également la variété des effets que l'on peut attendre des nombreuses métaphores qui apparaissent à la surface des médias.

Les médias, revenons-y, ne font pas que relayer simplement les métaphores de la culture ambiante. A en juger par leur étonnante production en matière de métaphore et par la récurrence de certaines d'entre elles dans plusieurs secteurs de l'information, on peut supposer qu'ils ont généré un système caractéristique de représentation métaphorique -et sans doute aussi métonymique.

Prenons un exemple. Dans une recherche portant sur le rapport entre les médias -principalement la télévision- et la constitution individuelle de la mémoire politique, Jérôme Bourdon a pu observer la tendance des spectateurs à conceptualiser métaphoriquement ce qui n'est que partiellement compris par eux. Dans la mémoire des sujets interrogés, la politique est surtout faite de grands événements (l'assassinat de Kennedy, Mai 68...) et ces événements sont appréhendés à travers une dramaturgie où dominent la fête et le drame et dans laquelle peu de place est faite à la politique elle-même, trop compliquée pour la plupart des spectateurs :

"Comme le notait Hoggart, comme le notent tant de mes enquêtes, «nous sommes envahis par une foule d'abstractions». Or il est une façon simple (...) de réordonner la vie politique. Les deuils, les ruptures, les retours: tous ces événements politiques sont ceux d'un long drame familial, devenu une allégorie du monde politique. Les membres des classes populaires ont conscience d'avoir accédé à un monde 
qu'ils se savent mal armés pour comprendre. On conçoit que, dans ces conditions, ils appliquent au monde extérieur les critères qu'ils utilisent pour juger et comprendre leur petit monde familier"1.

Bien entendu, cette métaphorisation du politique par les classes populaires ne résulte pas de l'action unilatérale de ces classes. C'est, dans une large mesure, la télévision elle-même qui organise métaphoriquement les événements.

"Les termes mêmes utilisés par la presse sont bien souvent ceux d'une métaphore familiale. Ainsi, la coexistence d'un premier ministre de droite et d'un président de gauche en 1986-88 est tout de suite devenue la «cohabitation», ce qui rendait immédiate une appréhension affective d'un problème que les politologues disséquaient par ailleurs avec délice. Cette allégorie familiale constitue un exemple de l'emploi de systèmes métaphoriques dans des contextes sociaux donnés..."2.

L'allégorie familiale -ou d'autres métaphores comme celle du complot également observée par J. Bourdon- a donc pour fonction de donner une certaine cohérence au savoir politique des spectateurs, mais au prix de quelle transformation et avec quelles conséquences sur les capacités d'action des citoyens?

Examinons un autre exemple de processus de métaphorisation observable dans l'information et particulièrement dans l'information politique.

En octobre-novembre 1992, en Belgique, le parti Écolo négocie avec le premier ministre son "oui" aux accords institutionnels de la Saint-Michel. Voici quelques expressions relevées dans la presse à cette époque, principalement dans les titres de trois grands quotidiens de la capitale:

- Le Soir du 29 octobre:

- "Après trois semaines de surplace, le bras de fer avec la majorité a véritablement démarré".

- "Dans la négociation du jour, le rapport de force est défavorable à Écolo...".

- "Certes les Verts le [Dehaene] font lanterner. Mais les dés paraissent pipés...".

1 J. Bourdon, "Télévision et symbolique politique", in Hermès, $\mathrm{n}^{\circ} 11-12,1993$, p. 203.

2 lbidem, p. 203. 
- "Les «Verts» ont riposté à l'offensive des patrons et des industries qui (...) tirent à vue sur le projet de lever des écotaxes".

- Le Soir du 31 octobre/1er novembre:

- "Dernier baroud pour refinancer le non-marchand. Écolo avait mission «d'aller le plus loin possible»... avant de déposer les armes...".

- "Dernier face-à-face".

- "Dehaene, dans la nuit de mercredi à jeudi, a rallongé la mise".

- "Sauf coup de théâtre, ce dernier bras de fer majorité-Ecolo... risquait peu d'aboutir à la rupture".

- "Mais à l'évidence, les négociateurs, même déçus par le refinancement, feront tout pour convaincre leurs troupes de ne pas lâcher la proie pour l'ombre".

- Le Soir du 2 novembre:

—“Samedi, à l'aube, Écolo a déposé les armes conscient d'avoir poussé la majorité dans ses derniers retranchements budgétaires".

Que retenir de ces trois semaines de face-à-face majoritéÉcolo? Parmi d'autres, le complet isolement des Verts, fusillés par l'opposition libérale...".

— "De même le couple Écolo-Agalev n'a pas résisté à ces trois semaines de bras de fer...".

- "Or, PS et PSC ne pouvaient batailler sur ce.terrain...".

— "Écolo a joué finement...".

- Le Soir du 5 novembre:

- "Divorce ou querelle d'amoureux entre Écolo et Agalev?"

- "Écolo dit oui: Dehaene a gagné" (gros titre).

- La Libre Belgique du 2 novembre:

- "Les Verts diront, dans quelques jours, s'ils se contentent d'un gain bien modeste ou s'ils préfèrent rejouer la mise au risque de tout perdre".

- La Dernière Heure du 29 octobre:

- "Jean-Luc Dehaene a enclenché le grand braquet. Comme prévu, il a entamé (...) la dernière ligne droite".

- La Dernière Heure du 6 novembre:

— "Les Écolos cocufiés" (sous-titre).

— "En apportant son soutien à un gouvernement rejeté par les trois quarts des Belges, Écolo vient de commettre une faute terrible froidement et sans circonstances atténuantes (...). Le masque est tombé. Soucieux d'accélérer leur arrivée au pouvoir, les Écolos ont signé un pacte avec Jean-Luc Dehaene prêt à tout pour sauver sa tête..."

Plusieurs métaphores inspirent tous ces énoncés: la métaphore guerrière, qui semble être la plus importante, la métaphore du jeu, la 
métaphore familiale et même une métaphore criminelle ("faute terrible", "circonstance atténuante"...).

Ces métaphores, du reste, se relient intérieurement. La guerre est souvent métaphorisée comme jeu et les rapports familiaux comportent une dimension conflictuelle qui justifie l'emploi de métaphores guerrières à leur sujet. Il s'agit au fond d'un véritable système métaphorique combinant plusieurs images s'impliquant l'une l'autre, le centre du système -son point fixe ou si l'on veut, son "attracteur", pour utiliser une métaphore empruntée à la physique- étant constitué par l'image de la guerre.

On peut voir ce système à l'œuvre dans une bonne partie de l'information politique, avec des degrés variables de saillance, bien sûr, en fonction de divers facteurs, dont sans doute le niveau de sensationnalisme.

Il ne fait pas de doute qu'un tel système poursuit des fins relationnelles -accroche du lecteur, recherche de complicité...- et confère au politique l'attrait du spectacle. Mais quelles peuvent être ses conséquences cognitives? On peut écarter d'emblée l'idée que la métaphore guerrière déclenche la réflexion et apporte un éclairage conceptuel nouveau en rendant visible un aspect jusqu'ici ignoré de la discussion entre politiques. Car il n'y a rien de nouveau ou d'incongru dans la métaphore. C'est très habituellement que l'on perçoit le débat politique en termes guerriers. Le système métaphorique (discussion politique $=$ guerre $=$ jeu...) est plutôt la manifestation d'une structuration conceptuelle profonde et stable. On trouvera difficilement d'autres systèmes métaphoriques dans l'information sur la politique. On trouvera même difficilement quelque chose qui corresponde à un sens propre par rapport auquel l'image de la guerre fasse figure de sens figuré. On peut dire que les médias ne font là que reprendre la métaphore commune "la discussion = la guerre", profondément enracinée dans notre culture selon Lakoff et Johnson. Mais il faut dire aussi que dans le cadre de la médiatisation des rapports politiques, la métaphore guerrière s'est considérablement consolidée. Et l'on peut comprendre pourquoi. L'image joue comme un opérateur de mise en spectacle. Elle a pour effet de ne retenir du débat politique que ceux de ses aspects qui se prêtent à la mise en récit. Ce qui compte, en effet, dans la métaphore guerrière, ce sont moins les problèmes politiques à résoudre que le jeu des personnages et leurs actions, leurs victoires et leurs échecs, bref tout ce qui comporte un 
aspect spectaculaire capable de captiver l'attention et de tenir en haleine. Figurons cela par un schéma:

Système métaphorique:

Discussion politique $=$

Guerre $=$

Mise en récit:

- personnages

- rapports de forces

Jeu... =

- succès, échec

La métaphore guerrière réduit notre savoir politique à une connaissance des rapports de force, des "stratégies" et des "tactiques" des "alliances" et "désunions", des "coups portés" et des "coups perdus", de la bataille qui se déroule dans l'arène politique. Elle réduit d'autant la connaissance des problèmes discutés et les capacités d'action du public, le confinant dans un rôle de spectateur des personnages mis en scène.

On peut objecter à cela qu'après tout, le système métaphorique fondé sur la guerre ne fait simplement que refléter la scène politique et les jeux qui s'y déroulent. Ce sont les politiques qui se comportent comme des stratèges guerriers. C'est vrai, mais justement, ne peut-on envisager que la représentation métaphorique n'agisse comme une contrainte sur le comportement des politiques? Il faut ici mettre en doute une conception bien ancrée dans l'opinion, conception selon laquelle les médias ne feraient que représenter des faits existant indépendamment d'eux, une réalité préexistante qu'ils ne feraient que refléter. En fait, entre les médias et la réalité, entre le représentant et le représenté, la relation est plutôt à concevoir sur le modèle de la circularité ou, si l'on veut, de la spécification réciproque. Les médias et le monde sont inclus l'un dans l'autre. Les rapports politiques entrânent des représentations métaphoriques qui à leur tour induisent des rapports politiques, cette boucle étant elle-même en relation de réciprocité avec l'arrière-plan culturel où elle s'inscrit. Dès lors, sans qu'on puisse leur attribuer un pouvoir absolu, on sent néanmoins la force de contrainte des métaphores. On pourrait prendre la mesure de cette force en examinant le traitement qui est réservé à ceux qui ne s'y conforment pas. Notre exemple contient une indication -insistons sur le terme "indication"- à ce sujet: les Écolos dont un des objectifs était de faire autrement de la politique ont été, dans les textes journalistiques cités plus haut, littéralement enfermés dans la métaphore guer- 
rière; ce qui, de leur action que l'on craignait déviante, a été perçu comme se conformant au rôle prescrit par la métaphore guerrière a inspiré à certains journalistes des propos qui ressemblent à des cris de victoire ou des soupirs de soulagement ("Écolo a mis la main dans un engrenage fatal. Adieu les grands principes, bonjour les compromissions, la stratégie, la course aux strapontins", La Dernière Heure du 6 novembre).

Tout cela nous conduit très loin de la métaphore-ornement du discours, jeu verbal destiné à amuser et séduire, attirer l'attention sur la réalité reflétée par les médias comme par un miroir. Les médias font partie de la réalité et les systèmes métaphoriques qu'ils utilisent contribuent à la structuration de notre connaissance du monde et par là du monde lui-même. Il importe d'en prendre conscience.

Une simple suggestion, naïve, pour terminer, concernant l'information politique: essayons d'imaginer une culture politique et médiatique où le débat politique n'est pas vu -ou moins- en termes de guerre, où il n'y a ni gagnant ni perdant, où attaquer ou défendre, gagner ou perdre du terrain, n'ont aucune signification... Essayons d'imaginer une culture politique où le débat politique est perçu comme un art ou une science, où les participants seraient moins considérés pour leurs qualités de combattants que pour leur coup d'œil et leur adresse dans l'art de comprendre -et de faire comprendre- les enjeux et la complexité des problèmes, où les électeurs seraient moins amenés à prendre parti dans une bataille qu'à participer à la compréhension de ces problèmes et à l'élaboration de solution...

\section{Conclusion}

L'idée principale qui se dégage des lignes précédentes et que l'on voudrait ici mettre bien en relief concerne la relation entre médias et société. Il faut remettre en cause le présupposé généralement admis qui voit dans les médias une sorte de miroir chargé de refléter, le plus objectivement possible, une réalité qui leur resterait extérieure. Ce présupposé n'est pas complètement faux, mais il masque certains aspects importants des choses. Il faut changer 
d'attitude de recherche et explorer l'idée de circularité1: les événements et leur récit, les rapports sociaux et leur représentation dans la presse, bref, les médias et la société, sont dans une relation de détermination réciproque ou, pour mieux dire, de coproduction. Dans cette optique, il faut convenir que les systèmes sémiotiques des médias (langages, formes d'énonciation, formes de narration...) coproduisent les réalités qu'elles reproduisent. Ces systèmes sont à la fois des structures d'accueil et de mise en forme de la réalité.

Parmi ces systèmes sémiotiques, la métaphore conçue moins sous son aspect poétique, lié au langage, que sous son aspect cognitif, lié à la pensée et à l'action. Il semble que dans leur commerce avec la société, les médias ont cristallisé quelques grands systèmes de métaphorisation largement récurrents. Ces systèmes conforment nos représentations et par là informent la réalité. C'est ce qu'illustre bien notre exemple -qu'il faudrait évidemment développer-emprunté à la communication politique: la discussion politique et sa représentation au moyen d'un jeu cohérent de métaphores sont dans une relation de codépendance ou de conformité réciproque qui lie l'une à l'autre l'activité politique et l'activité du journaliste. Cette relation est sans doute d'autant plus contraignante que les systèmes métaphoriques en question sont implicites, inaperçus, stabilisés, profondément inscrits dans les manières habituelles (professionnalisées) de parler et d'écrire les faits. La métaphore, cependant, peut tout aussi bien ouvrir la boucle de la codépendance lorsqu'elle s'exhibe en tant que telle et surtout lorsque sa nouveauté décentre l'attention et enclenche la réflexion sur des aspects ignorés des choses.

1 La notion de circularité s'est formée dans le courant de la pensée systémique. On peut en suivre le développement dans le cadre des sciences humaines chez des auteurs comme Bateson, Watzlawick, Morin, Barel, Varela. 